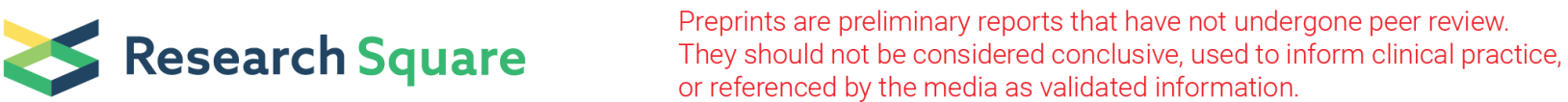

\section{Characterizing the structural covariance network in AD-susceptible single nucleotide polymorphisms and the correlations with cognitive outcomes}

\section{Hsin-I Chang}

Chang Gung Memorial Hospital Kaohsiung Branch

Yu-Tzu Chang

National Cheng Kung University Medical Center: National Cheng Kung University Hospital

Chi-Wei Huang

Chang Gung Memorial Hospital Kaohsiung Branch

Kuo-Lun Huang

Chang Gung Memorial Hospital Linkou Main Branch: Chang Gung Memorial Hospital

Jung-Lung Hsu

Chang Gung Memorial Hospital Linkou Main Branch: Chang Gung Memorial Hospital

Shih-Wei Hsu

Chang Gung Memorial Hospital Kaohsiung Branch

Shih-Jen Tsai

National Yang Ming University Hospital

Wen-Neng Chang

Chang Gung Memorial Hospital Kaohsiung Branch

Chen-Chang Lee

Chang Gung Memorial Hospital Kaohsiung Branch

Shu-Hua Huang

Chang Gung Memorial Hospital Kaohsiung Branch

Chiung-Chih Chang ( $\nabla$ neur099@adm.cgmh.org.tw)

Chang Gung Memorial Hospital Kaohsiung Branch https://orcid.org/0000-0002-6721-2556

\section{Research article}

Keywords: Alzheimer disease, single nucleotide polymorphism, independent component analysis

Posted Date: September 28th, 2020

DOI: https://doi.org/10.21203/rs.3.rs-71595/v1

License: (a) (7) This work is licensed under a Creative Commons Attribution 4.0 International License. Read Full License 


\section{Abstract}

Background

The clinical manifestations of Alzheimer disease $(A D)$ are related to brain network degeneration, while genetic differences may mediate network change patterns. A number of AD-susceptible loci have been reported using genome-wide association studies, however, how they modulate intracerebral volume and relationships to cognitive outcomes remains to be established. We hypothesized that different genotype groups may modulate large-scale brain networks independently or interact with apolipoprotein E4 (ApoE4) status to determine neurobehavior test scores.

Methods

Gray matter structural covariance networks were constructed in 324 patients with $A D$ using $T 1$ magnetic resonance imaging with independent component analysis (ICA). We assessed 15 genetic loci (rs9349407, rs3865444, rs670139, rs744373, rs3851179, rs11136000, rs3764650, rs610932, rs6887649, rs7849530, rs4866650, rs3765728, rs34011, rs6656401, rs597668) using the additive, recessive and dominant model on clinical outcomes. Statistical analysis was performed to explore the independent role of each locus, interactions with ApoE4 status, and relationships to brain ICA network integrity score. We used Cognitive Abilities Screening Instrument (CASI) total score or short-term memory (STM) subscore as the major outcome factors, adjusted for covariates of education, disease duration and age.

Results

Clinically, the CD2APG allele showed a protective role in CASI-total and CASI-STM scores independently or via interactions with non-ApoE4 status, while the CR1 A genotype group was associated with lower STM independently of ApoE4 status. Three loci showed synergic interactions with ApoE4: BIN $1 T$ allele and MS4A6A G allele with non-ApoE4 status, and FTMTG allele with ApoE4 status. The network integrity scores revealed 9 significant ICA networks (anterior and posterior hippocampus, right temporal, right or left thalamus, inferior cerebellum, medial cerebellum, default mode network, frontal attention network) that correlated with cognitive scores, in which only the ApoE4 and $M S 4 A 6 A$ genotype group was independently related to the hippocampus network. Genetic loci of MS4A6A, BIN1, CD2AP, CD33, CLU, BIN1, P73, EXOC3L2, CR1 and MS4AE4 exerted network influence independently or via interactions with ApoE4 status.

Conclusions

This study suggests that AD-susceptible loci may exert clinical significance independently, through interactions with ApoE4 status or by modulating ICA networks to determine cognitive outcomes.

\section{Background}

Alzheimer disease $(A D)$ is a complex disease. For late-onset $A D$, the apo-lipoprotein $E(A p o E)$ gene represents the most significant example of a single nucleotide polymorphism (SNP) that modifies AD susceptibility (Singh, Singh et al. 2006). The clinical significance of SNPs on brain integrity has been shown in studies exploring the effect of ApoE4 allele in people with AD (Martins, Oulhaj et al. 2005), those with mild cognitive impairment (Risacher, Kim et al. 2013), and non-demented elderly (Bretsky, Guralnik et al. 2003, Wisdom, Callahan et al. 2011). The presence of the ApoE4 allele (Corder, Saunders et al. 1993) remains the greatest risk factor for $A D$ (Farrer, Cupples et al. 1997), responsible for a 3- to 15-fold increase in risk. The synergic effect of the ApoE4 allele with other genetic loci has been reported in previous studies (Mahley 1988), which showed that overall pathological cascades may influence the neuropsychiatric outcomes (Hall, Wiechmann et al. 2014). Although genetic effects have been extensively studied, only a few have shown reproducible results.

Recent large-scale genome-wide association studies (GWASs) have revealed a number of SNPs other than ApoE4 that are also associated with AD susceptibility. A total of 19 GWAS loci have been identified using a discovery sample of 17,008 AD patients and 37,154 controls, followed by replication with 8,572 AD patients and 11,312 controls (Lambert, Ibrahim-Verbaas et al. 2013). In 2019, a GWAS meta-analysis reported an additional 29 risk loci (Jansen, Savage et al. 2019). Collectively termed as AD susceptible genes, they can only explain a small proportion of the heritability and influence of SNPs for risk prediction, and the results have been inconsistent among different ethnicities. For example, the rs 111360000 polymorphism in the clusterin ( $C L U$ ) gene has been found to be significantly associated with AD (Zhu, Liu et al. 2018) in Caucasian and Asian populations, but not people of African or Hispanic descent (Du, Tan et al. 2016). The genetic-intracerebral interactions may be different among different ethnicities, and so the genetic effect on cognitive outcomes may also be different. For AD, structural imaging is considered to be the most robust tool to reveal correlations with cognitive measures, while links between reported genotype groups and neuroimaging biomarkers may help to understand the intracerebral modulations of these SNPs. The clinical significance of these risk loci and their interactions with brain networks may also help to understand the neurobiology of AD.

Recent research suggests that highly related regions show covariance in morphometric characteristics, so-called structural covariance. Structural covariance networks (SCNs) have been associated with structural and functional connectivity, while genetic variations, developmental, degenerative and disease staging have been shown to be important covariates of interest (Alexander-Bloch, Giedd et al. 2013). The SCN is considered to be a reproducible and heritable model that may represent disease-related or genetic-associated changes in topology (Huang, Hsu et al. 2017, Chang, Chang et al. 2018, Chang, Tsai et al. 2018, Chang, Chang et al. 2019). In theory, AD susceptible genes may have greater covariance in gray matter (GM). Using spatial independent component analysis (ICA), the structural covariance between topographically distant regions can be modeled without a priori knowledge (Biswal, Yetkin et al. 1995, Biswal, Mennes et al. 2010). In addition to fully automated spatial component maps of maximal statistical independence maps, a network integrity score can be calculated for each ICA map. The network integrity score describes the strength of the individual expression in each network, and a higher network integrity score indicates stronger individual expression of the identified network (Beckmann and Smith 2004, Segall, Allen et al. 2012). Once an SCN pattern has been identified to distinguish one group from another, its expression can be prospectively quantified on an individual basis and correlated with the clinical or physiological 
measures of interest. The SNP-brain relationships modelled by ICA can be used to test the co-variation of GM between genotype groups, while the network integrity score can be used as a dependent variable to test the genetic interactions.

In this study, we tested whether SCNs constructed using ICA could serve as an endophenotype for cognitive test outcomes in AD patients. Based on GWAS results, we assessed 15 AD-associated SNPs belonging to the following genetic loci: CD2AP (rs9349407), CD33 (rs3865444), MS4AE4 (rs670139), BIN1 (rs744373), PICALM (rs3851179), CLU (rs11136000), ABCA7(rs3764650), MS4A6A (rs610932), FTMT (rs6887649), SPTLC1 (rs7849530), Intergenic SNP (rs4866650), p73 (rs3765728), FGF1 (rs34011), CR1 (rs6656401) and EXOC3L2 (rs597668). We tested the independent effect of each SNP on cognitive outcomes and evaluated whether the associations were related to interactions with the ApoE4 genotype. The clinical significance of individual SNPs or interactions between the SNP and ApoE4 on SCN was calculated and validated by correlating the network integrity score and cognitive scores.

\section{Methods}

This study was conducted in accordance with the Declaration of Helsinki and was approved by the Institutional Review Board of Chang Gung Memorial Hospital. The study participants were treated at the Cognition and Aging Center, Department of General Neurology, Kaohsiung Chang Gung Memorial Hospital. The multi-disciplinary team was composed of behavior neurologists, psychiatrists, neuropsychologists, neuroradiologists and experts in nuclear medicine. We enrolled patients with AD who were diagnosed according to the International Working Group-2 criteria (Dubois, Feldman et al. 2014), and further confirmed by amyloid imaging (TW-ADNI: http://tadni.cgmh-mi.com/home) if the consensus panel did not agree on the diagnosis. All of the patients were in a stable condition under acetylcholine esterase inhibitor treatment from the time of diagnosis. The exclusion criteria were a past history of clinical stroke, a negative amyloid scan, a modified Hachinski ischemic score $>4$ and depression. After checking the inclusion and exclusion criteria, a total of 324 subjects ( 152 males, 172 females) were included and underwent imaging and genetic tests.

\section{Clinical and Neurobehavioral Assessments}

After enrolment, the demographic data of each patient were recorded. We also recorded the time of the first symptom. A trained neuropsychologist administered the neurobehavioral tests using Cognitive Abilities Screening Instrument (CASI) total scores as a global assessment of cognitive function. The CASI contains nine subdomains. We used attention, verbal fluency, abstract thinking, and mental manipulation subdomain scores to assess executive function (Huang, Chang et al. 2013), and orientation, short- and long-term memory, language ability, and drawing as non-executive domains. As the salient feature of $A D$ is short-term memory (STM) impairment, we used the CASI-STM subscore as the major outcome for genetic correlations.

\section{Genotyping}

SNP genotyping was performed using MassARRAY technology with iPLEX Gold chemistry (Agena Bioscience, San Diego, CA, USA). The PCR primers and single-base-extension primers were designed using Assay Design Suite v2.0 software. The genotyping analysis was performed using an iPLEX Gold Reagent Kit according to the manufacturer's instructions. Briefly, $1 \mu \mathrm{l}$ of DNA sample $(10 \mathrm{ng} / \mu \mathrm{l})$ was subjected to $5 \mu$ of PCR reaction containing 0.2 units of Taq polymerase, $2.5 \mathrm{pmol}$ each of the PCR primers and $25 \mathrm{mM}$ each of the dNTPs. Thermocycling was started at $94^{\circ} \mathrm{C}$ for 2 min followed by $45 \mathrm{cycles}$ of $94^{\circ} \mathrm{C}$ for $30 \mathrm{~s}, 56^{\circ} \mathrm{C}$ for $30 \mathrm{~s}$ and $72^{\circ} \mathrm{C}$ for $1 \mathrm{~min}$, and a final extension was done at $72^{\circ} \mathrm{C}$ for $1 \mathrm{~min}$. Unincorporated dNTPs were dephosphorylated by $0.3 \mathrm{U}$ of shrimp alkaline phosphatase. Purified amplicons were then subjected to primer extension using an iPLEX Gold Reagent Kit. Primer extension was performed with a cycling program of $94^{\circ} \mathrm{C}$ for $30 \mathrm{~s}$, followed by 40 cycles of $94^{\circ} \mathrm{C}$ for $5 \mathrm{~s}$, and 5 cycles of $52^{\circ} \mathrm{C}$ for $5 \mathrm{~s}$ and $80^{\circ} \mathrm{C}$ for $5 \mathrm{~s}$ within 40 cycles, followed by a final extension at $72^{\circ} \mathrm{C}$ for $3 \mathrm{~min}$. The extended reaction products were purified by cation exchange resins and then spotted onto a 384-format SpectroCHIP II array using a MassArray Nanodispenser RS1000. Mass determination was done on a MassARRAY Compact Analyzer. The resulting spectra were processed and alleles called with MassARRAY Typer 4.0 with model-based cluster analysis to analyze the genotypes of the SNPs. We tested 15 SNPs (rs9349407, rs3865444, rs670139, rs744373, rs3851179, rs11136000, rs3764650, rs610932, rs6887649, rs7849530, rs4866650, rs3765728, rs34011, rs6656401, rs597668). The risk alleles and minor allele frequencies (MAFs) are listed in Supplementary Table 1. The ApoE genotype was determined using rs7412 and rs429358. ApoE4 carriers were defined as those with one or two E4 alleles.

\section{Image Acquisition}

Magnetic resonance images were acquired using a 3.0T magnetic resonance imaging (MRI) scanner (Excite, GE Medical Systems, Milwaukee, WI, USA). All MRI images were performed within 3 months of cognitive test scores. High-resolution structural images were acquired for spatial normalization using the following protocols: a T1-weighted, inversion-recovery-prepared, three-dimensional, gradient-recalled acquisition in a steady-state sequence with a repetition time/echo time/inversion time of $8,600 \mathrm{~ms} / \mathrm{minimal} / 450 \mathrm{~ms}$, a $256 \times 256 \mathrm{~mm}$ field of view, and a 1-mm slice sagittal thickness with a resolution of $0.5 \times 0.5 \times$ $1 \mathrm{~mm} 3$.

\section{Data Analysis for Neuroimaging Biomarkers}

Image preprocessing and statistical analysis were performed using SPM12 (Wellcome Trust Centre of Cognitive Neurology, University College London, UK, http://www.fil.ion.ucl.ac.uk/spm/). The T1 images were reoriented, realigned, and normalized using the standard Montreal Neurological Institute space. The images were then segmented into GM and white matter. Related tissue segments were used to create a custom template using the diffeomorphic anatomical registration using exponentiated lie algebra approach, which is one of the highest ranking registration methods in patients with $A D$ (Cuingnet, Gerardin et al. 2011). The modulated and warped images were then smoothed using a Gaussian kernel of $8 \mathrm{~mm}$ full width at half maximum.

The preprocessed spatial normalized modified T1 images from the patients were concatenated to form a subject series and entered into the ICA process. Spatial ICA was carried out using Multivariate Exploratory Linear Optimized Decomposition into Independent Components software package version 3.15 (http://fsl.fmrib.ox.ac.uk/fsl/fslwiki/MELODIC). The resulting independent components were $z$-transformed and visualized using a threshold of $z>1.96$ ( $p<$ 
0.05). Differences in ICA intensities between groups in each SNP were calculated. In addition, interactions between individual SNPS and ApoE4 status were also modelled using a general linear model, corrected for age, educational level, gender and disease duration (years). To understand the clinical significance of the identified SCN in the patients, we also calculated partial correlations between the extracted SCN intensity and the clinical scores by setting the significance value at $p<0.05$ using Bonferroni correction for multiple comparisons and covariates of age, educational level and disease duration (years).

\section{Statistical Analysis}

Clinical and laboratory data were expressed as mean \pm standard deviation. The Student's $t$ test was used to compare continuous variables, and the chi-square test was used for categorical variables. Linear regression models of associations between the cognitive scores and 15 selected AD-related SNPs were analyzed, adjusting for education, age and duration of disease. All statistical analyses were conducted using SPSS software (SPSS version 22 for Windows $\AA_{\text {, }}$ SPSS Inc., Chicago, IL). Statistical significance was set at $p<0.05$.

\section{Results}

\section{Demographic Data of the AD Patients}

The demographic data and cognitive test scores are shown in Table 1. The mean duration of disease was $0.9 \pm 1.12$ years, and 114 cases were E4 carriers (E4 homozygote $\mathrm{n}=14$ ). The risk alleles of $C D 2 A P, A B C A 7, F T M T, S P T L C 1, F G F 1, C R 1$ and EXOC3L2 were found on the minor alleles (Supplementary Table 1). Of note, the MAFs of FTMT and CR1 were extremely low.

Table 1

Demographic data of the 324 patients with Alzheimer disease

\begin{tabular}{|ll|}
\hline & Case numbers or scores \\
\hline Male/Female & $152 / 172$ \\
\hline Education (year) & $7.8 \pm 5.09$ \\
\hline Duration of disease (year) & $0.9 \pm 1.12$ \\
\hline ApoE4 carriers (\%) & $114(35.19 \%)$ \\
\hline Age (years) & $71.6 \pm 8.82$ \\
\hline Cognition Ability Screening Instrument & \\
\hline Total score (100) & $68.6 \pm 24.68$ \\
\hline Executive function test (40) & $25.5 \pm 9.62$ \\
\hline Attention (8) & $6.3 \pm 1.63$ \\
Verbal fluency (10) & $5.4 \pm 3.08$ \\
Abstract thinking (12) & $8.1 \pm 3.12$ \\
Mental manipulation (10) & $5.7 \pm 3.50$ \\
\hline Non-executive domains (60) & $43.2 \pm 15.83$ \\
\hline Orientation (18) & $13.0 \pm 5.71$ \\
Short- term memory (12) & $6.1 \pm 4.11$ \\
Long-term memory (10) & $8.3 \pm 2.42$ \\
Language ability (10) & $8.1 \pm 2.42$ \\
Drawing (10) & $7.7 \pm 2.99$ \\
\hline Data presented as mean \pm standard deviation. Numbers in & parentheses indicate maximal score. \\
\hline
\end{tabular}

\section{Effect Of Independent Genotype Groups On Cognitive Outcomes}

Two SNPs showed independent or synergic effects on cognitive measures. The CD2APG allele was associated with CASI-total scores (Table 2) on the additive or dominant model, and the $\mathrm{G}$ allele was associated with higher scores. The $C D 2 A P \mathrm{G}$ allele (on the additive or dominant model) was also associated with a higher STM score (Table 3). The association between the CD2APG allele and cognitive scores showed interactions with the non-ApoE4 genotype in CASI-total score (Table 2) and STM score (Table 3). In the recessive model, the CR1 A allele was associated with a lower STM score (Table 3), while the risk of a detrimental effect was independent of ApoE4 status. Of note, the MAF of the A allele in CR1 was 0.029 . 
Table 2

General linear model for single nucleotide polymorphisms on cognitive ability screening instrument total scores*

\begin{tabular}{|c|c|c|c|c|c|c|c|c|c|c|c|c|c|}
\hline \multirow[t]{2}{*}{ Gene } & \multicolumn{4}{|l|}{ Additive model } & \multicolumn{3}{|c|}{ Dominant model } & \multicolumn{3}{|c|}{ Recessive Model } & \multicolumn{3}{|c|}{ Interaction with APOE } \\
\hline & Group & $\beta$ & SE & $\mathrm{p}$ & $\beta$ & SE & $\mathrm{p}$ & $\beta$ & SE & $\mathrm{p}$ & $\begin{array}{l}\text { Chi- } \\
\text { square }\end{array}$ & $\mathrm{p}$ & effects \\
\hline$C D 2 A P$ & $\begin{array}{l}\text { GG compares to } \\
\text { CC }(1 / 3)\end{array}$ & 44.091 & 14.17 & 0.002 & 43.245 & 14.165 & 0.002 & -4.337 & 3.0951 & 0.161 & 5.989 & $\hat{0}_{0.05}$ & $\begin{array}{l}\text { Non- } \\
\text { E4 }\end{array}$ \\
\hline rs9349407 & $\begin{array}{l}\text { CG compares to } \\
\text { CC }(2 / 3)\end{array}$ & 41.125 & 14.32 & 0.004 & & & & & & & & & \\
\hline CD33 & $\begin{array}{l}\text { CC compares to } \\
\text { AA }(1 / 3)\end{array}$ & 0.406 & 7.658 & 0.958 & 1.048 & 7.5987 & 0.890 & 1.746 & 2.9576 & 0.555 & 2.489 & 0.477 & - \\
\hline rs 3865444 & $\begin{array}{l}\text { CA compares to } \\
\text { AA }(2 / 3)\end{array}$ & 2.398 & 7.8728 & 0.761 & & & & & & & & & \\
\hline MS4AE4 & $\begin{array}{l}\text { GG compares to } \\
\text { TT }(1 / 3)\end{array}$ & 2.26 & 4.2595 & 0.596 & 4.152 & 3.8605 & 0.282 & 1.896 & 2.9644 & 0.522 & 6.002 & 0.112 & - \\
\hline s670139 & $\begin{array}{l}\text { GT compares to } \\
\text { TT }(2 / 3)\end{array}$ & 5.514 & 4.0691 & 0.175 & & & & & & & & & \\
\hline BIN1 & $\begin{array}{l}\text { AA compares to } \\
\text { GG }(1 / 3)\end{array}$ & 0.089 & 4.4880 & 0.984 & 1.080 & 4.1155 & 0.793 & 1.32 & 2.941 & 0.654 & 3.612 & 0.306 & - \\
\hline rs744373 & $\begin{array}{l}\text { AG compares to } \\
\mathrm{GG}(2 / 3)\end{array}$ & 1.791 & 4.3096 & 0.678 & & & & & & & & & \\
\hline PICALM & $\begin{array}{l}\text { CC compares to } \\
\text { TT }(1 / 3)\end{array}$ & -3.254 & 4.1484 & 0.433 & -2.431 & 3.7963 & 0.522 & 1.986 & 2.9024 & 0.494 & 3.117 & 0.374 & - \\
\hline rs3851179 & $\begin{array}{l}\text { CT compares to } \\
\text { TT }(2 / 3)\end{array}$ & -1.733 & 4.0528 & 0.669 & & & & & & & & & \\
\hline$C L U$ & $\begin{array}{l}\text { CC compares to } \\
\text { TT }(1 / 3)\end{array}$ & 0.490 & 6.8294 & 0.943 & 1.801 & 6.7691 & 0.790 & 3.333 & 2.9119 & 0.252 & 2.972 & 0.396 & - \\
\hline rs11136000 & $\begin{array}{l}\text { TC compares to } \\
\text { TT }(2 / 3)\end{array}$ & 4.358 & 7.0434 & 0.536 & & & & & & & & & \\
\hline$A B C A 7$ & $\begin{array}{l}\text { TT compares to } \\
\text { GG }(1 / 3)\end{array}$ & 0.127 & 4.4213 & 0.977 & 1.915 & 4.1498 & 0.644 & 2.657 & 2.8650 & 0.354 & 3.034 & 0.386 & - \\
\hline rs3764650 & $\begin{array}{l}\text { TG compares to } \\
\mathrm{GG}(2 / 3)\end{array}$ & 3.636 & 4.4014 & 0.409 & & & & & & & & & \\
\hline$M S 4 A 6 A$ & $\begin{array}{l}\text { GG compares to } \\
\text { TT }(1 / 3)\end{array}$ & -1.407 & 4.2567 & 0.741 & 0.329 & 3.8545 & 0.932 & 2.588 & 2.9492 & 0.380 & 4.623 & 0.202 & - \\
\hline rs10932 & $\begin{array}{l}\text { TG compares to } \\
\text { TT }(2 / 3)\end{array}$ & 1.562 & 4.0599 & 0.7 & & & & & & & & & \\
\hline FTMT & $\begin{array}{l}\text { AA compares to } \\
\text { GG }(1 / 3)\end{array}$ & 26.705 & 24.7683 & 0.281 & 26.328 & 24.7763 & 0.288 & 2.905 & 3.8570 & 0.451 & 2.337 & 0.126 & - \\
\hline rs6887649 & $\begin{array}{l}\text { AG compares to } \\
\mathrm{GG}(2 / 3)\end{array}$ & 24.296 & 24.9768 & 0.331 & & & & & & & & & \\
\hline SPTLC1 & $\begin{array}{l}\text { AA compares to } \\
\text { GG }(1 / 3)\end{array}$ & 11.770 & 12.4733 & 0.345 & 11.596 & 12.4562 & 0.352 & 1.663 & 3.5659 & 0.641 & 2.738 & 0.434 & - \\
\hline rs7849530 & $\begin{array}{l}\text { AG compares to } \\
\mathrm{GG}(2 / 3)\end{array}$ & 10.829 & 12.8078 & 0.398 & & & & & & & & & \\
\hline $\begin{array}{l}\text { Intergenic } \\
\text { SNP }\end{array}$ & $\begin{array}{l}\text { AA compares to } \\
\text { CC }(1 / 3)\end{array}$ & -4.298 & 8.0343 & 0.593 & -4.654 & 7.9625 & 0.559 & -0.533 & 2.9044 & 0.854 & 3.990 & 0.263 & - \\
\hline rs4866650 & $\begin{array}{l}\mathrm{AC} \text { compares to } \\
\mathrm{CC}(2 / 3)\end{array}$ & -5.279 & 8.1853 & 0.519 & & & & & & & & & \\
\hline P73 & $\begin{array}{l}\text { GG compares to } \\
\text { AA }(1 / 3)\end{array}$ & -0.260 & 4.2196 & 0.951 & 1.091 & 2.9254 & 0.709 & 1.076 & 3.9070 & 0.783 & 3.319 & 0.345 & - \\
\hline rs3765728 & $\begin{array}{l}\text { GA compares to } \\
\mathrm{AA}(2 / 3)\end{array}$ & 1.606 & 3.1456 & 0.610 & & & & & & & & & \\
\hline FGF1 & $\begin{array}{l}\text { GG compares to } \\
\text { AA }(1 / 3)\end{array}$ & -6.776 & 4.5323 & 0.144 & -5.592 & 4.4370 & 0.208 & 3.584 & 2.8329 & 0.206 & 5.267 & 0.153 & - \\
\hline
\end{tabular}

*adjusted for education, disease duration and age

SE: standard error; 


\begin{tabular}{|c|c|c|c|c|c|c|c|c|c|c|c|c|c|}
\hline \multirow{2}{*}{$\begin{array}{l}\text { Gene } \\
\text { rs34011 }\end{array}$} & \multicolumn{4}{|l|}{ Additive model } & \multicolumn{3}{|c|}{ Dominant model } & \multicolumn{3}{|c|}{ Recessive Model } & \multicolumn{3}{|c|}{ Interaction with $\mathrm{APOE}$} \\
\hline & $\begin{array}{l}\text { GA compares to } \\
\text { AA }(2 / 3)\end{array}$ & -4.123 & 4.7375 & 0.384 & & & & & & & & & \\
\hline CR1 & $\begin{array}{l}\text { AA compares to } \\
\text { GG }(1 / 3)\end{array}$ & 34.971 & 24.7119 & 0.157 & 34.67 & 24.7429 & 0.161 & -7.488 & 6.1687 & 0.225 & 3.999 & 0.135 & - \\
\hline rs6656401 & $\begin{array}{l}\text { GA compares to } \\
\mathrm{GG}(2 / 3)\end{array}$ & -5.771 & 6.3347 & 0.362 & & & & & & & & & \\
\hline EXOC3L2 & $\begin{array}{l}\text { TT compares to } \\
\text { CC }(1 / 3)\end{array}$ & -2.806 & 4.3082 & 0.515 & -4.447 & 3.9970 & 0.266 & -1.65 & 2.8892 & 0.568 & 2.783 & 0.426 & - \\
\hline rs597668 & $\begin{array}{l}\text { TC compares to } \\
\operatorname{CC}(2 / 3)\end{array}$ & -5.899 & 4.2413 & 0.164 & & & & & & & & & \\
\hline \multicolumn{14}{|c|}{ *adjusted for education, disease duration and age } \\
\hline SE: standa & & & & & & & & & & & & & \\
\hline
\end{tabular}


Table 3

General linear model for single nucleotide polymorphisms on cognitive ability screening instrument short-term memory score*

\begin{tabular}{|c|c|c|c|c|c|c|c|c|c|c|c|c|c|}
\hline \multirow[t]{2}{*}{ Gene } & \multicolumn{4}{|l|}{ Additive model } & \multicolumn{3}{|c|}{ Dominant model } & \multicolumn{3}{|c|}{ Recessive Model } & \multicolumn{3}{|c|}{ Interaction with APOE } \\
\hline & Group & $\beta$ & SE & $\mathrm{p}$ & $\beta$ & SE & $\mathrm{p}$ & $\beta$ & SE & $\mathrm{p}$ & $\begin{array}{l}\text { Chi- } \\
\text { square }\end{array}$ & $\mathrm{p}$ & effects \\
\hline$C D 2 A P$ & $\begin{array}{l}\text { GG compares to CC } \\
(1 / 3)\end{array}$ & 5.596 & 2.3663 & 0.018 & 5.418 & 2.3673 & 0.022 & -0.790 & 0.5135 & 0.124 & 7.950 & 0.019 & $\begin{array}{l}\text { Non- } \\
\text { E4 }\end{array}$ \\
\hline rs9349407 & $\begin{array}{l}\text { CG compares to } \\
\text { CC }(2 / 3)\end{array}$ & 4.971 & 2.3903 & 0.038 & & & & & & & & & \\
\hline CD33 & $\begin{array}{l}\text { CC compares to AA } \\
(1 / 3)\end{array}$ & 1.086 & 1.2685 & 0.392 & 1.225 & 1.2594 & 0.331 & 0.277 & 0.4910 & 0.573 & 5.597 & 0.133 & - \\
\hline rs 3865444 & $\begin{array}{l}\text { CA compares to } \\
\mathrm{AA}(2 / 3)\end{array}$ & 1.518 & 1.3042 & 0.244 & & & & & & & & & \\
\hline MS4AE4 & $\begin{array}{l}\text { GG compares to TT } \\
(1 / 3)\end{array}$ & 0.506 & 0.7078 & 0.475 & 0.675 & 0.6407 & 0.292 & 0.095 & 0.4923 & 0.847 & 6.163 & 0.104 & - \\
\hline s670139 & $\begin{array}{l}\text { GT compares to TT } \\
(2 / 3)\end{array}$ & 0.797 & 0.6762 & 0.239 & & & & & & & & & \\
\hline BIN1 & $\begin{array}{l}\text { AA compares to GG } \\
(1 / 3)\end{array}$ & 0.488 & 0.7432 & 0.511 & 0.238 & 0.6819 & 0.727 & -0.442 & 0.4869 & 0.364 & 7.7977 & 0.046 & E4 \\
\hline rs744373 & $\begin{array}{l}\text { AG compares to } \\
\mathrm{GG}(2 / 3)\end{array}$ & 0.059 & 0.7136 & 0.934 & & & & & & & & & \\
\hline PICALM & $\begin{array}{l}\text { CC compares to TT } \\
(1 / 3)\end{array}$ & 0.035 & 0.6895 & 0.960 & 0.087 & 0.6308 & 0.890 & 0.061 & 0.4823 & 0.899 & 6.756 & 0.080 & - \\
\hline rs3851179 & $\begin{array}{l}\text { CT compares to TT } \\
(2 / 3)\end{array}$ & 0.132 & 0.6736 & 0.845 & & & & & & & & & \\
\hline$C L U$ & $\begin{array}{l}\text { CC compares to TT } \\
(1 / 3)\end{array}$ & 0.024 & 1.1313 & 0.983 & 0.268 & 1.1236 & 0.811 & 0.758 & 0.4824 & 0.116 & 6.739 & 0.081 & - \\
\hline rs11136000 & $\begin{array}{l}\text { TC compares to TT } \\
(2 / 3)\end{array}$ & 0.837 & 1.1667 & 0.473 & & & & & & & & & \\
\hline$A B C A 7$ & $\begin{array}{l}\text { TT compares to GG } \\
(1 / 3)\end{array}$ & 1.112 & 0.7320 & 0.129 & 1.159 & 0.6856 & 0.091 & -0.190 & 0.4760 & 0.690 & 6.606 & 0.086 & - \\
\hline rs3764650 & $\begin{array}{l}\text { TG compares to } \\
\text { GG }(2 / 3)\end{array}$ & 1.204 & 0.7287 & 0.098 & & & & & & & & & \\
\hline$M S 4 A 6 A$ & $\begin{array}{l}\text { GG compares to TT } \\
(1 / 3)\end{array}$ & 0.509 & 0.7056 & 0.470 & -0.117 & 0.6389 & 0.855 & 0.632 & 0.4888 & 0.196 & 8.070 & 0.045 & $\begin{array}{l}\text { Non- } \\
\text { E4 }\end{array}$ \\
\hline rs10932 & $\begin{array}{l}\text { TG compares to TT } \\
(2 / 3)\end{array}$ & 0.162 & 0.6730 & 0.809 & & & & & & & & & \\
\hline FTMT & $\begin{array}{l}\text { AA compares to GG } \\
(1 / 3)\end{array}$ & 5.961 & 4.1087 & 0.147 & -5.954 & 4.1075 & 0.147 & 0.161 & 0.6410 & 0.802 & 4.598 & 0.032 & E4 \\
\hline rs6887649 & $\begin{array}{l}\text { AG compares to } \\
\mathrm{GG}(2 / 3)\end{array}$ & 5.921 & 4.1433 & 0.153 & & & & & & & & & \\
\hline SPTLC1 & $\begin{array}{l}\text { AA compares to GG } \\
(1 / 3)\end{array}$ & 1.145 & 2.0743 & 0.581 & 1.198 & 2.0720 & 0.563 & 0.188 & 0.5928 & 0.751 & 6.861 & 0.076 & - \\
\hline rs7849530 & $\begin{array}{l}\text { AG compares to } \\
\mathrm{GG}(2 / 3)\end{array}$ & 1.429 & 2.1299 & 0.502 & & & & & & & & & \\
\hline $\begin{array}{l}\text { Intergenic } \\
\text { SNP }\end{array}$ & $\begin{array}{l}\text { AA compares to CC } \\
(1 / 3)\end{array}$ & 0.597 & 1.3297 & 0.653 & -0.853 & 1.3220 & 0.519 & -0.594 & -0.4811 & 0.217 & 5.203 & 0.158 & - \\
\hline rs4866650 & $\begin{array}{l}\text { AC compares to } \\
\mathrm{CC}(2 / 3)\end{array}$ & 1.302 & 1.3547 & 0.337 & & & & & & & & & \\
\hline P73 & $\begin{array}{l}\text { GG compares to AA } \\
(1 / 3)\end{array}$ & 0.545 & 0.7934 & 0.438 & -0.424 & 0.4876 & 0.385 & 0.353 & 0.6516 & 0.588 & 6.753 & 0.080 & - \\
\hline rs3765728 & $\begin{array}{l}\text { GA compares to } \\
\mathrm{AA}(2 / 3)\end{array}$ & 0.378 & 0.5244 & 0.471 & & & & & & & & & \\
\hline FGF1 & $\begin{array}{l}\text { GG compares to AA } \\
(1 / 3)\end{array}$ & 0.372 & 0.7702 & 0.629 & -0.299 & 0.7369 & 0.685 & 0.212 & 0.4705 & 0.653 & 4.551 & 0.208 & - \\
\hline
\end{tabular}

*adjusted for education, disease duration and age

SE: standard error; 


\begin{tabular}{|c|c|c|c|c|c|c|c|c|c|c|c|c|c|}
\hline \multirow{2}{*}{$\begin{array}{l}\text { Gene } \\
\text { rs34011 }\end{array}$} & \multicolumn{4}{|l|}{ Additive model } & \multicolumn{3}{|c|}{ Dominant model } & \multicolumn{3}{|c|}{ Recessive Model } & \multicolumn{3}{|c|}{ Interaction with $\mathrm{APOE}$} \\
\hline & $\begin{array}{l}\text { GA compares to } \\
\text { AA }(2 / 3)\end{array}$ & 0.208 & 0.7877 & 0.792 & & & & & & & & & \\
\hline CR1 & $\begin{array}{l}\text { AA compares to GG } \\
(1 / 3)\end{array}$ & 5.587 & 4.0857 & 0.171 & 5.483 & 4.1096 & 0.182 & -2.217 & 1.0189 & 0.030 & 4.670 & 0.198 & - \\
\hline rs6656401 & $\begin{array}{l}\text { GA compares to } \\
\mathrm{GG}(2 / 3)\end{array}$ & 2.006 & 1.0473 & 0.055 & & & & & & & & & \\
\hline EXOC3L2 & $\begin{array}{l}\text { TT compares to CC } \\
(1 / 3)\end{array}$ & 0.131 & 0.7166 & 0.855 & -0.115 & 0.6647 & 0.862 & -0.382 & 0.4793 & 0.425 & 7.655 & 0.054 & - \\
\hline rs597668 & $\begin{array}{l}\text { TC compares to } \\
\text { CC }(2 / 3)\end{array}$ & 0.333 & 0.7055 & 0.637 & & & & & & & & & \\
\hline \multicolumn{14}{|c|}{ *adjusted for education, disease duration and age } \\
\hline SE: standar & & & & & & & & & & & & & \\
\hline
\end{tabular}

Effect of Interactions with the ApoE4 Gene on STM Scores

Three SNPs showed interactions with the ApoE4 gene: BIN 1, MS4A6A and FTMT. The risk allele of BIN1 T allele and FTMT G allele showed interactions with the E4 allele, while the MS4A6A G allele showed a protective role via interactions with non-E4 alleles. The MAF of the G allele in FTMT was 0.082 .

\section{Network Topography And Clinical Significance Validation}

To understand the effects of SCN and SNP genotype groups, a total of 20 ICA components were constructed. For each SNP, we compared network integrity score between SNP genotype groups (Supplementary Table 2). The stratification of each SNP genotype group was based on the number of cases and the balance of Hardy-Weinberg equilibrium. The spatial extent of 15 ICA modes showing genotypic differences is shown in Fig. 1.

Using network integrity scores, we correlated each significant ICA with cognitive tests (Table 4) to establish network significance. The CASI scores were classified as indicating general cognitive performance (CASI-total score), executive domains and non-executive domains. The anterior hippocampus, posterior hippocampus, right temporal, right thalamus, left thalamus, inferior cerebellum default mode network and frontal attention network were significantly related to CASI-total scores. For correlations with executive domains scores, the network patterns overlapped with the general performance network except for default mode network and inferior cerebellum. The topography of non-executive networks overlapped with the CASI-total score network. Meanwhile, the network integrity scores of supplementary motor area (SMA) and postcentral gyrus were inversely correlated with STM. 
Significant relationships between independent component analysis and cognitive test scores

\begin{tabular}{|c|c|c|c|c|c|c|c|c|c|c|}
\hline \multicolumn{2}{|l|}{ Area } & \multirow[t]{2}{*}{ Gene and SNP } & \multirow{2}{*}{$\begin{array}{l}\text { CASI } \\
\text { Total } \\
\text { scores }\end{array}$} & \multicolumn{4}{|c|}{ Executive Domains } & \multicolumn{3}{|c|}{ Non-executive Domains } \\
\hline $\begin{array}{l}\text { Index } \\
\text { On } \\
\text { Figure } \\
1\end{array}$ & $\begin{array}{l}\text { Network Main } \\
\text { Structure }\end{array}$ & & & Attention & $\begin{array}{l}\text { Verbal } \\
\text { fluency }\end{array}$ & $\begin{array}{l}\text { Abstract } \\
\text { thinking }\end{array}$ & $\begin{array}{l}\text { Mental } \\
\text { manipulation }\end{array}$ & Orientation & $\begin{array}{l}\text { Short- } \\
\text { term } \\
\text { memory }\end{array}$ & $\begin{array}{l}\text { Long- } \\
\text { term } \\
\text { memory }\end{array}$ \\
\hline \multirow[t]{3}{*}{ A } & \multirow[t]{3}{*}{ Basal Ganglia } & CLU (Rs11136000) & \multirow[t]{3}{*}{-0.063} & \multirow[t]{3}{*}{-0.036} & \multirow[t]{3}{*}{-0.096} & \multirow[t]{3}{*}{-0.094} & \multirow[t]{3}{*}{-0.069} & \multirow[t]{3}{*}{-0.023} & \multirow[t]{3}{*}{-0.072} & \multirow[t]{3}{*}{-0.035} \\
\hline & & FGF1 (rs34011) & & & & & & & & \\
\hline & & $\begin{array}{l}\text { APOE4 * PICALM } \\
\text { (rs3851179) }\end{array}$ & & & & & & & & \\
\hline \multirow[t]{4}{*}{ B } & \multirow{4}{*}{$\begin{array}{l}\text { Anterior } \\
\text { Hippocampus }\end{array}$} & APOE & \multirow[t]{4}{*}{$0.315(* *)$} & \multirow[t]{4}{*}{0.084} & \multirow[t]{4}{*}{$0.121(*)$} & \multirow[t]{4}{*}{$0.188(* *)$} & \multirow[t]{4}{*}{$0.222(* \star)$} & \multirow[t]{4}{*}{$0.387(\star \star)$} & \multirow[t]{4}{*}{$0.365(* *)$} & \multirow[t]{4}{*}{$0.280(* *)$} \\
\hline & & $\begin{array}{l}\text { APOE * MS4AE4 } \\
\text { (rs670139) }\end{array}$ & & & & & & & & \\
\hline & & $\begin{array}{l}\text { APOE * MS4A6A } \\
\text { (rs610932) APOE * } \\
\text { P73 (rs3765728) }\end{array}$ & & & & & & & & \\
\hline & & $\begin{array}{l}\text { APOE * CR1 } \\
\text { (rs6656401) }\end{array}$ & & & & & & & & \\
\hline \multirow[t]{8}{*}{ C } & \multirow{8}{*}{$\begin{array}{l}\text { Posterior } \\
\text { Hippocampus }\end{array}$} & APOE & \multirow[t]{8}{*}{$0.434(* *)$} & \multirow[t]{8}{*}{$0.217(* *)$} & \multirow[t]{8}{*}{$0.338(* \star)$} & \multirow[t]{8}{*}{$0.279(* *)$} & \multirow[t]{8}{*}{$0.262(* *)$} & \multirow[t]{8}{*}{$0.460(* *)$} & $0.492(* *)$ & $0.314(* \star)$ \\
\hline & & MS4A6A (rs610932) & & & & & & & & \\
\hline & & $\begin{array}{l}\text { APOE * MS4AE4 } \\
\text { (rs670139) }\end{array}$ & & & & & & & & \\
\hline & & $\begin{array}{l}\text { APOE * BIN1 } \\
\text { (rs744373) }\end{array}$ & & & & & & & & \\
\hline & & $\begin{array}{l}\text { APOE * } \\
\text { PICALM(rs3851179) }\end{array}$ & & & & & & & & \\
\hline & & $\begin{array}{l}\text { APOE * MS4A6A } \\
\text { (rs610932) }\end{array}$ & & & & & & & & \\
\hline & & $\begin{array}{l}\text { APOE * CR1 } \\
\text { (rs6656401) }\end{array}$ & & & & & & & & \\
\hline & & $\begin{array}{l}\text { APOE * EXOC3L2 } \\
\text { (rs597668) }\end{array}$ & & & & & & & & \\
\hline D & Right & MS4A6A (rs610932) & $0.351(* *)$ & $0.177(* \star)$ & $0.233(* *)$ & $0.237(* *)$ & $0.275(* \star)$ & $0.337(* *)$ & $0.357(* *)$ & $0.300(* *)$ \\
\hline & Teimporai & $\begin{array}{l}\text { APOE * FTMT } \\
\text { (rs6887649) }\end{array}$ & & & & & & & & \\
\hline E & $\begin{array}{l}\text { Right } \\
\text { Thalamus }\end{array}$ & MS4A6A (rs610932) & $0.167(* *)$ & $0.128(*)$ & $0.138(*)$ & $0.192(* *)$ & 0.075 & 0.129 & $0.123(*)$ & $0.113(*)$ \\
\hline $\mathbf{F}$ & Left Thalamus & BIN1 (rs744373) & $0.128(*)$ & $0.116(*)$ & $0.115(*)$ & $0.157(* *)$ & 0.058 & 0.105 & $0.126\left(^{*}\right)$ & 0.051 \\
\hline
\end{tabular}




\begin{tabular}{|c|c|c|c|c|c|c|c|c|c|c|}
\hline \multirow{3}{*}{$\begin{array}{l}\text { Area } \\
\text { G }\end{array}$} & \multirow{3}{*}{$\begin{array}{l}\text { Postcentral } \\
\text { Gyrus }\end{array}$} & \multirow{2}{*}{$\begin{array}{l}\text { Gene and SNP } \\
\text { APOE }\end{array}$} & \multirow{2}{*}{$\begin{array}{l}\text { CASI } \\
-0.082\end{array}$} & \multicolumn{2}{|c|}{ Executive Domains } & \multirow[b]{2}{*}{-0.020} & \multirow[b]{2}{*}{-0.028} & \multicolumn{2}{|c|}{ Non-executive Domains } & \multirow[b]{2}{*}{-0.033} \\
\hline & & & & -0.005 & -0.106 & & & -0.109 & $-0.121(*)$ & \\
\hline & & CD2AP (rs9349407) & & & & & & & & \\
\hline & & $\begin{array}{l}\text { APOE * CD2AP } \\
\text { (rs9349407) }\end{array}$ & & & & & & & & \\
\hline & & $\begin{array}{l}\mathrm{APOE} * \mathrm{CD} 33 \\
(\mathrm{rs} 3865444)\end{array}$ & & & & & & & & \\
\hline & & $\begin{array}{l}\text { APOE * MS4AE4 } \\
\text { (rs670139) }\end{array}$ & & & & & & & & \\
\hline & & $\begin{array}{l}\text { APOE * } \\
\text { PICALM(rs3851179) }\end{array}$ & & & & & & & & \\
\hline & & $\begin{array}{l}\text { APOE *ABCA7 } \\
\text { (rs3764650) }\end{array}$ & & & & & & & & \\
\hline & & $\begin{array}{l}\text { APOE * MS4A6A } \\
\text { (rs610932) }\end{array}$ & & & & & & & & \\
\hline & & $\begin{array}{l}\text { APOE * SPTLC1 } \\
\text { (rs7849530) }\end{array}$ & & & & & & & & \\
\hline & & $\begin{array}{l}\text { APOE * P73 } \\
\text { (rs3765728) }\end{array}$ & & & & & & & & \\
\hline & & $\begin{array}{l}\text { APOE * CR1 } \\
\text { (rs6656401) }\end{array}$ & & & & & & & & \\
\hline & & $\begin{array}{l}\text { APOE * EXOC3L2 } \\
\text { (rs597668) }\end{array}$ & & & & & & & & \\
\hline \multirow[t]{11}{*}{ H } & \multirow{11}{*}{$\begin{array}{l}\text { Supplementary } \\
\text { Motor Cortex }\end{array}$} & APOE & -0.072 & -0.016 & -0.074 & -0.071 & -0.074 & -0.085 & -0.114 & -0.029 \\
\hline & & CD33 (rs3865444) & & & & & & & & \\
\hline & & CLU (rs11136000) & & & & & & & & \\
\hline & & P73 (rs3765728) & & & & & & & & \\
\hline & & $\begin{array}{l}\text { APOE * CD33 } \\
\text { (rs3865444) }\end{array}$ & & & & & & & & \\
\hline & & $\begin{array}{l}\text { APOE * CLU } \\
\text { (rs11136000) }\end{array}$ & & & & & & & & \\
\hline & & $\begin{array}{l}\text { APOE * MS4AE4 } \\
\text { (rs670139) }\end{array}$ & & & & & & & & \\
\hline & & $\begin{array}{l}\text { APOE * MS4A6A } \\
\text { (rs610932) }\end{array}$ & & & & & & & & \\
\hline & & $\begin{array}{l}\text { APOE * P73 } \\
\text { (rs3765728) }\end{array}$ & & & & & & & & \\
\hline & & $\begin{array}{l}\text { APOE * FGF1 } \\
\text { (rs34011) }\end{array}$ & & & & & & & & \\
\hline & & $\begin{array}{l}\text { APOE * EXOC3L2 } \\
\text { (rs597668) }\end{array}$ & & & & & & & & \\
\hline I & $\begin{array}{l}\text { Inferior } \\
\text { Cerebellum }\end{array}$ & BIN1 (rs744373) & $0.126\left(^{*}\right)$ & -0.084 & -0.093 & -0.069 & -0.051 & $-0.136\left(^{*}\right)$ & $-0.136\left(^{*}\right)$ & $-0.163(* \star)$ \\
\hline$J$ & $\begin{array}{l}\text { Lateral } \\
\text { Cerebellum }\end{array}$ & $\begin{array}{l}\text { SPTLC1 } \\
\text { (rs7849530, } \\
\text { rs4866650) }\end{array}$ & -0.015 & 0.029 & 0.022 & 0.043 & -0.035 & -0.043 & -0.030 & -0.018 \\
\hline K & $\begin{array}{l}\text { Medial } \\
\text { Cerebellum }\end{array}$ & $\begin{array}{l}\text { EXOC3L2 } \\
\text { (rs597668) }\end{array}$ & $0.151(* *)$ & -0.080 & $-0.131\left(^{*}\right)$ & -0.110 & -0.079 & $-0.150(* *)$ & -0.096 & $-0.178(* \star)$ \\
\hline \multirow[t]{2}{*}{ L } & \multirow{2}{*}{$\begin{array}{l}\text { Default Mode } \\
\text { Network }\end{array}$} & CLU (rs11136000) & $-0.117(*)$ & 0.096 & 0.088 & 0.072 & 0.086 & $0.150(* \star)$ & $0.143(*)$ & 0.051 \\
\hline & & BIN1 (rs744373) & & & & & & & & \\
\hline \multirow[t]{2}{*}{ M } & \multirow{2}{*}{$\begin{array}{l}\text { Frontal } \\
\text { Attention } \\
\text { Network }\end{array}$} & CR1 (rs6656401) & $0.211(* *)$ & $0.164(\star *)$ & $\begin{array}{l}0.198 \\
(* *)\end{array}$ & $0.186(* *)$ & $0.142(*)$ & $0.191(* \star)$ & $0.176(\star *)$ & $0.115(*)$ \\
\hline & & $\begin{array}{l}\text { APOE * EXOC3L2 } \\
\text { (rs597668) }\end{array}$ & & & & & & & & \\
\hline
\end{tabular}




\begin{tabular}{|c|c|c|c|c|c|c|c|c|c|c|}
\hline \multirow{2}{*}{$\begin{array}{l}\text { Area } \\
\mathrm{N}\end{array}$} & \multirow[b]{2}{*}{ Cerebellum } & \multirow{2}{*}{$\begin{array}{l}\text { Gene and SNP } \\
\text { MS4AE4 (rs670139) }\end{array}$} & \multirow{2}{*}{$\begin{array}{l}\text { CASI } \\
-0.063\end{array}$} & \multicolumn{4}{|c|}{ Executive Domains } & \multicolumn{3}{|c|}{ Non-executive Domains } \\
\hline & & & & -0.040 & $-0.145\left(^{*}\right)$ & -0.032 & -0.040 & -0.059 & -0.030 & -0.029 \\
\hline 0 & $\begin{array}{l}\text { Cingulate } \\
\text { Network }\end{array}$ & ABCA7 (rs3764650) & -0.041 & 0.060 & 0.067 & 0.006 & 0.011 & 0.041 & 0.030 & 0.049 \\
\hline
\end{tabular}

Numbers under the cognitive test name represent partial correlation coefficient, adjusted for age, disease duration and education.

\section{Network Effect for ApoE4, Preselected SNP and Genetic-ApoE4 Interactions}

Significant differences between ApoE4 and non-E4 carriers in network intensity scores were identified in anterior and posterior hippocampus (Fig. 1B, 1C), postcentral gyrus (Fig. 1G) and SMA (Fig. 1H).

A number of networks showed SNP-ApoE4 interactions (Table 4, Fig. 1A-D, G, H, M). These networks included cardinal networks of AD, including anterior and posterior hippocampus, right temporal, postcentral gyrus, frontal executive network and supplementary motor cortex. All preselected SNPs interacted with ApoE4 status in the abovementioned networks except CLU. Among the 15 networks, only basal ganglia (Fig. $1 \mathrm{~A}$ ), lateral cerebellum (Fig. $1 \mathrm{~J}$ ) and cingulate network (Fig. 10) showed no correlations with clinical scores.

A number of SNPs exerted a network influence independently from ApoE4, including MS4A6A, BIN1, CD2AP, CD33, CLU, BIN1, P73, EXOC3L2, CR1 and MS4AE4. Independent roles were observed for CLU on basal ganglia, SMA and default mode network (Fig. 1A, 1H, 1L), FGF1 on basal ganglia (Fig. 5A), MS4A6A on hippocampus and temporal, thalamus (Fig. 5C-E), BIN1 on left thalamus, cerebellum, default mode network (Fig. 1F, 1I, 1L), CD2AP on post-central gyrus (Fig. 1G), CD33 and $p 73$ on SMA (Fig. 1H), CR1 on frontal attention network (Fig. 1M), and ABCA7 on cingulate network (Fig. 10).

\section{Discussion}

\section{Major Findings}

In this study, we explored the clinical significance of 15 AD-susceptible loci by constructing SCNs using an ICA approach in patients with AD. Our results reflected complex interactions among preselected SNPs, brain network integrity and cognitive outcomes in which the mechanisms were via different pathways. First, the SCN served as the endophenotype in predicting individual genotype groups in cognitive outcomes. For SCN as the endophenotype, the genotype group either exerted an independent role or synergistic interactions with ApoE4 status on cognitive measures. The SCNs showing clinical significance included the hippocampal axis, temporal, thalamus, default mode network and frontal attention network. As the SCNs were each associated with different cognitive domains, the identification of genotype-SCN relationships may help to understand the neurobiology in AD. Second, for genetic-clinical relationships, our results suggested that the main genotype effect on cognitive measures was via interactions with ApoE4 status. This included protective $C D 2 A P \mathrm{G}$ allele and MS4A6A G allele with non-ApoE4 interactions, and risk allele of BIN1 T allele and FTMT G allele with ApoE4 status. Of note, independent roles of genotype groups ( $C D 2 A P$ and $C R 1)$ on cognitive outcomes were also found. However, as $C R 1$ risk allele A was a minor allele with a low MAF, the $C R 1$ finding here should be interpreted with caution.

\section{ApoE4 Modulated Hippocampal SCN and Determined the Salient Feature of AD}

By definition, the SCN is based on the similarity of the same microstructural variations and thus may be influenced by factors influencing underlying structures such as the expression of common genetic traits during development. The independent role of ApoE4 on the hippocampal and SMA SCN was established in this study. As the hippocampal SCN intensity score was also correlated with STM score in our AD patients, this finding supports the role of ApoE4 in linking salient cognitive and biosignature features in $A D$.

Traditional cytoarchitectonic distribution of the hippocampus (Frederickson, Klitenick et al. 1983) is a convoluted GM structure encompassing three architectonically distinct regions: the fascia dentata, the CA region (which can be subdivided into CA1-CA4 fields), and the subicular complex. In this study, two hippocampus SCNs in ICA (anterior versus posterior hippocampus) showed ApoE4 genotype group differences. The SCN of the hippocampus axis associated with ApoE4 showing the anterior-posterior axis is consistent with task-related activities or connectivity patterns (Colombo, Fernandez et al. 1998, Przezdzik, Faber et al. 2019). A similar anterior-posterior organization has recently been reported, suggesting that the gene expression is linked to the distinct molecular gradient in the hippocampus (Vogel, La Joie et al. 2020). Among the 15 preselected SNPs and ApoE4, only ApoE4 showed an independent role in the entire hippocampal axis. The association with cognitive test scores in hippocampal SCN intensity scores and the interactions between hippocampus and cortical/subcortical structures demonstrate its tight integration within the large-scale degenerative system, so that hippocampus-ApoE4 is the most important factor in predicting $A D$ functional severity.

\section{ApoE4 Interacted with MS4A, CR1, PICALM, p73, BIN1 and EXOC3L2 on the Hippocampal Axis}

Our results showed the role of MS4A6A (rs610932) on posterior hippocampus axis. In addition, MS4A4E and MSA4A6A also interacted with ApoE4 in the entire hippocampal SCN, similar to that reported in a smaller AD cohort (Chang, Mori et al. 2019). A previous study reported interactions between MS4A and CLU (Lambert, Ibrahim-Verbaas et al. 2013) or CD33 on conferring the risk of AD. Common variants of MS4A6A (rs610932), along with MS4A4E (rs670139), CD33 (rs3865444) and CD2AP(rs9349407), CLU and PICALM have been associated with memory decline (Hollingworth, Harold et al. 2011, Naj, Jun et al. 2011, Karch, Jeng et al. 2012). Our results emphasize the role of the hippocampus and MS4A epistasis on the clinical features of AD. 
The SNPs that interacted with the ApoE4 allele on the hippocampal axis in this study may explain the mechanisms of AD susceptibility. CR1 on chromosome 1 (rs6656401, odds ratio [OR] = 1.21, 95\% confidence interval [Cl] 1.14-1.29, $\mathrm{p}=3.7 \times 10^{-9}$ ) has been shown to encode complement component (3b/4b) receptor 1 (Lambert, Heath et al. 2009) which is known to participate in the clearance of $\beta$ amyloid peptides in AD pathology. By facilitating the clearance of $\mathrm{A} \beta$ peptides through enhanced translocation, increased micro-vessel PICALM (phosphatidylinositol binding clathrin assembly protein) (rs3851179, OR $=0.86$, $p=1.3 \times 10^{-9}$ ) has been shown to reduce the development of AD (Harold, Abraham et al. 2009). The SNP rs3765728 within tumor protein p73 (Tao, Sun et al. 2014) has been associated with neuron survival, and has been found to have a synergetic effect with APOE on the risk of AD. In addition, rs744373 near BIN1 $\left(\mathrm{OR}=1.13 ; 95 \% \mathrm{Cl}, 1.06-1.21\right.$ per copy of the minor allele; $\left.\mathrm{p}=1.59 \times 10^{-11}\right)$ has been related to $A D$ with possible mechanisms involving tau-related cascade (Franzmeier, Rubinski et al. 2019). Moreover, rs597668 is near EXOC3L2 (OR, 1.18; $\left.95 \% \mathrm{Cl}, 1.07-1.29 ; \mathrm{p}=6.45 \times 10^{-9}\right)$, and mutations of this gene may be associated with AD.

\section{Dominant Model Suggested Protection of the CD2AP rs9349407 G Allele in Non-E4 Carriers}

The role of rs9349407 as a risk SNP in Han Chinese people has been reported (Xiao, Liu et al. 2015), but the findings have been inconsistent (Jiao, Liu et al. 2015). One meta-analysis suggested that rs9349407 C is a risk allele for AD susceptibility in East Asian, American, Canadian, and European populations (Chen, Wu et al. 2015). In our additive or dominant model, AD patients with the rs $9349407 \mathrm{G}$ allele had higher CASI total and STM scores, especially the nonE4 carriers. The minor allele C of rs 9349407 has been associated with neuritic plaque burden in pathology, which may explain why non-E4 carriers with the $\mathrm{G}$ allele may have higher cognitive test scores (Shulman, Chen et al. 2013).

In the current study, we only enrolled patients with clinical AD, and we tested whether risk or protective SNP alleles have an effect on cognitive test scores or SCNs. Although not all of our patients with AD received amyloid scans, our exclusion criteria reduced the possibility of non-AD pathologies. rs9349407 is a polymorphism in the $C D 2 A P$ gene which translates the scaffolding molecule for signal transduction. Loss of function of $C D 2 A P$ has been linked to enhanced $A \beta$ production, tau-induced neurotoxicity, abnormal neurite structure modulation and reduced blood-brain barrier integrity, which has been implicated in $A D$ pathogenesis (Dubey, Gulati et al. 2018, Ramos de Matos, Ferreira et al. 2018).

\section{Modulation of Frontal Attention Network by the CR1 rs6656401 A Allele on Cognitive Outcomes}

As STM is the salient feature in AD, it was used as a dependent variable in SNP genetic model analysis. In addition to rs9349407, we also identified the independent role of rs6656401 A allele on lower STM scores, consistent with a previous meta-analysis of a greater risk on the minor allele A in AD (Shen, Chen et al. 2015). CR1 is an AD susceptibility locus that also influences AD-related traits on neuritic plaque deposition and in episodic memory decline. The coding variant in the long homologous repeat $\mathrm{D}$ region of the $C R 1$ gene, rs4844609 (Ser1610Thr) has been associated with episodic memory decline and accounts for the known effect of SNP rs6656401 (Keenan, Shulman et al. 2012). In SCN analysis in this study, the effect may have been partially modulated by interactions with ApoE4 on the hippocampal axis.

Three studies have confirmed the association between AD susceptibility and the rs6656401 A allele in Chinese patients (Zhang, Yu et al. 2010, Chen, Kao et al. 2012, Jin, Li et al. 2012), although another study showed no association (Li, Shi et al. 2011). rs6656401 is in the CR1 gene, and complement system activation in the clearance of amyloid has been proposed to be a possible mechanism of the risk associated with rs6656401 (Zhang, Yu et al. 2010 ). Although the independent role of rs6656401 minor allele on lower STM was confirmed in this study, it is worth noting that the A allele was a minor allele and the MAF was only 0.029. Therefore, the relationship with lower STM may be due to the small sample size of the A allele. To understand the effect, we also checked SCN intensity and explored whether the relationships between the rs6656401 A allele and cognitive test results were modulated by SCN. As shown in our correlation analysis, the frontal attention SCN intensity was significantly different between A carriers and G allele, and the intensity was also related to the executive and non-executive domains. Therefore, we suggest that the frontal attention network is the endophenotype of the link between rs6656401 and cognitive outcomes.

\section{Effect of FTMT on STM in AD via ApoE4 and Default Mode Network}

The intergenic SNP (rs6887649) is $10 \mathrm{~KB}$ upstream of ferritin mitochondrial gene (FTMT), which has been shown to modify the association between amyloid positivity and baseline ventricular volume (Hohman, Koran et al. 2014). From our study, the effect of FTMT on STM is via interaction with ApoE4, while it also modulates the default mode network. The default mode network is regarded to be an early neuroimaging bio-signature (Chang, Huang et al. 2015), and a recent report suggested that the default mode network may be comprised of multiple, spatially dissociated but interactive components (Andrews-Hanna, Reidler et al. 2010), of which two subsystems are of particular interest. Our SCN result of an association with FTMT was consistent with cores in the posterior cingulate cortex and anterior medial prefrontal cortex that is known as the "dorsal medial prefrontal cortex subsystem" (or midline core subsystem).

\section{Limitations And Methodological Considerations}

This study has several limitations. First, we enrolled subjects with early stage AD, and we did not include a control group. As the SNPs were preselected from GWAS results showing significance in AD susceptibility, the inclusion of a control group may have helped to elucidate whether these SNPs exerted similar GM modulation patterns in healthy elderly subjects as in those with AD. However, as these SNPs each exert different functional activities on the pathogenetic mechanisms in $A D$, the use of a pure AD population may help to maximize the effect of each SNP on regional GM networks. As a structural covariance matrix is defined by estimating the inter-regional correlations of cortical volumes between all possible pairs of regions defined by anatomy, SCN construction relies on both the spatial patterns of morphometric and signal similarities. Given the differences in brain morphometry in controls, it would be difficult to match all influential factors. Therefore, we focused on the initial hypothesis testing the endophenotypic role of SCN in cognitive outcomes. Another limitation is the estimation of the number of components for ICA analysis. Most studies have used 12 to 30 components in structural networks or resting state networks. In this study, we constructed 20 networks and filtered the clinical significance of a network using correlation analysis with cognitive measures and by matching with the ICA template (https://brainmap.org/icns/). From a methodological aspect, the group-wise structural covariance analysis relied on the morphological 
properties of each voxel with the rest of the brain across a group of participants. The registration of a single participant's structural data to the template involves linear and non-linear deformation that can result in inaccuracies in subregions. In this report, we emphasized the well-characterized network to explain the SNP effect where the network significance was established by correlations with cognitive measures. Finally, the use of 15 AD-susceptible SNPs to validate the underlying pathological mechanisms may have oversimplified the genetic interactions. We only tested interactions between each SNP and ApoE4 status as ApoE4 remains the strongest predictor. The interpretations of interactions between SNPS and SCN were based on a literature review of possible alterations in functional pathways and may not fully explain the in vivo situation. Therefore, the findings should be interpreted with caution.

\section{Conclusion And Future Perspectives}

In this study, we aimed to elucidate the major modes of structure variation in AD-susceptible SNPs and assess the SNP co-plastic properties. Our findings demonstrated AD-related SNP effects that may influence the SCN independently or synergistically with ApoE4. The use of SCN as an endophenotype allowed us to assume the independent and synergistic role of putative SNPs to predict cognitive measures. The complex interplay among these SNPs in our study suggests that a hierarchical order of SNPs modulate brain networks.

\section{Abbreviations}

AD Alzheimer disease

ApoE apolipoprotein $\mathrm{E}$

CASI Cognitive Abilities Screening Instrument

GM gray matter

GWAS genome-wide association study

ICA independent component analysis

MAF minor allele frequency

MRI magnetic resonance imaging

SCN structural covariance network

SMA supplementary motor area

SNP single nucleotide polymorphism

STM short-term memory

\section{Declarations}

The authors have nothing to declare.

\section{Ethics approval and consent to participate}

This study was approved by the Chang Gung Memorial Hospital Ethics Committee following the standards for medical research in humans recommended by the Declaration of Helsinki. All participants gave signed, informed consent for the cognitive tests described, inclusion of samples, neuroimaging and demographic data.

\section{Consent for publication}

Not applicable

\section{Availability of data and materials}

The data analyzed during the current study are available from the corresponding author on reasonable request.

\section{Competing interests}

The authors declare that they have no competing interests.

\section{Funding}

The work was supported by the Ministry of Science Taiwan (MOST 109-2314-B-182A-082), Chang Gung Memorial Hospital (CMRPG8J0521, CMRPG8J0841) to $\mathrm{CCC}$. 


\section{Authors' contributions}

CWH, KLH, JLH, SWH, WNC, CCL, SHH and CCC contributed to the acquisition and analysis of data. HIC, YTC and CCC drafted a significant portion of the manuscript and figures. All authors read and approved the final manuscript.

\section{Acknowledgments}

We thank the participants and their families for their generosity. The authors thank the Genomics Center for Clinical and Biotechnological Applications of National Core Facility for Biopharmaceuticals, Taiwan (MOST 109-2740-B-010-002) for genotyping.

\section{References}

Alexander-Bloch, A., J. N. Giedd and E. Bullmore (2013). "Imaging structural co-variance between human brain regions." Nat Rev Neurosci14(5): 322-336.

Andrews-Hanna, J. R., J. S. Reidler, J. Sepulcre, R. Poulin and R. L. Buckner (2010). "Functional-anatomic fractionation of the brain's default network." Neuron65(4): 550-562.

Beckmann, C. F. and S. M. Smith (2004). "Probabilistic independent component analysis for functional magnetic resonance imaging." IEEE Trans Med Imaging23(2): 137-152.

Biswal, B., F. Z. Yetkin, V. M. Haughton and J. S. Hyde (1995). "Functional connectivity in the motor cortex of resting human brain using echo-planar MRI." Magn Reson Med34(4): 537-541.

Biswal, B. B., M. Mennes, X. N. Zuo, S. Gohel, C. Kelly, S. M. Smith, C. F. Beckmann, J. S. Adelstein, R. L. Buckner, S. Colcombe, A. M. Dogonowski, M. Ernst, D. Fair, M. Hampson, M. J. Hoptman, J. S. Hyde, V. J. Kiviniemi, R. Kotter, S. J. Li, C. P. Lin, M. J. Lowe, C. Mackay, D. J. Madden, K. H. Madsen, D. S. Margulies, H. S. Mayberg, K. McMahon, C. S. Monk, S. H. Mostofsky, B. J. Nagel, J. J. Pekar, S. J. Peltier, S. E. Petersen, V. Riedl, S. A. Rombouts, B. Rypma, B. L. Schlaggar, S. Schmidt, R. D. Seidler, G. J. Siegle, C. Sorg, G. J. Teng, J. Veijola, A. Villringer, M. Walter, L. Wang, X. C. Weng, S. Whitfield-Gabrieli, P. Williamson, C. Windischberger, Y. F. Zang, H. Y. Zhang, F. X. Castellanos and M. P. Milham (2010). "Toward discovery science of human brain function." Proc Natl Acad Sci U S A107(10): 4734-4739.

Bretsky, P., J. M. Guralnik, L. Launer, M. Albert and T. E. Seeman (2003). "The role of APOE-epsilon4 in longitudinal cognitive decline: MacArthur Studies of Successful Aging." Neurology60(7): 1077-1081.

Chang, C. C., Y. T. Chang, C. W. Huang, S. J. Tsai, S. W. Hsu, S. H. Huang, C. C. Lee, W. N. Chang, C. C. Lui and C. Y. Lien (2018). "Associations of Bcl-2 rs956572 genotype groups in the structural covariance network in early-stage Alzheimer's disease." Alzheimers Res Ther10(1): 17.

Chang, C. C., S. J. Tsai, N. C. Chen, C. W. Huang, S. W. Hsu, Y. T. Chang, M. E. Liu, W. N. Chang, W. C. Tsai and C. C. Lee (2018). "Catechol-O-Methyltransferase Val158Met Polymorphism on Striatum Structural Covariance Networks in Alzheimer's Disease." Mol Neurobiol55(6): 4637-4649.

Chang, H. I., Y. T. Chang, S. J. Tsai, C. W. Huang, S. W. Hsu, M. E. Liu, W. N. Chang, C. Y. Lien, S. H. Huang, C. C. Lee and C. C. Chang (2019). "MAOA-VNTR Genotype Effects on Ventral Striatum-Hippocampus Network in Alzheimer's Disease: Analysis Using Structural Covariance Network and Correlation with Neurobehavior Performance." Mol Neurobiol56(6): 4518-4529.

Chang, Y. T., C. W. Huang, Y. H. Chang, N. C. Chen, K. J. Lin, T. C. Yan, W. N. Chang, S. F. Chen, C. C. Lui, P. H. Lin and C. C. Chang (2015). "Amyloid burden in the hippocampus and default mode network: relationships with gray matter volume and cognitive performance in mild stage Alzheimer disease." Medicine (Baltimore).94(16): e763.

Chang, Y. T., E. Mori, M. Suzuki, M. Ikeda, C. W. Huang, J. J. Lee, W. N. Chang and C. C. Chang (2019). "APOE-MS4A genetic interactions are associated with executive dysfunction and network abnormality in clinically mild Alzheimer's disease." Neuroimage Clin21: 101621.

Chen, H., G. Wu, Y. Jiang, R. Feng, M. Liao, L. Zhang, G. Ma, Z. Chen, B. Zhao, K. Li, C. Yu and G. Liu (2015). "Analyzing 54,936 Samples Supports the Association Between CD2AP rs9349407 Polymorphism and Alzheimer's Disease Susceptibility." Mol Neurobiol52(1): 1-7.

Chen, L. H., P. Y. Kao, Y. H. Fan, D. T. Ho, C. S. Chan, P. Y. Yik, J. C. Ha, L. W. Chu and Y. Q. Song (2012). "Polymorphisms of CR1, CLU and PICALM confer susceptibility of Alzheimer's disease in a southern Chinese population." Neurobiol Aging33(1): 210 e211-217.

Colombo, M., T. Fernandez, K. Nakamura and C. G. Gross (1998). "Functional differentiation along the anterior-posterior axis of the hippocampus in monkeys." J Neurophysiol80(2): 1002-1005.

Corder, E. H., A. M. Saunders, W. J. Strittmatter, D. E. Schmechel, P. C. Gaskell, G. W. Small, A. D. Roses, J. L. Haines and M. A. Pericak-Vance (1993). "Gene dose of apolipoprotein E type 4 allele and the risk of Alzheimer's disease in late onset families." Science261(5123): 921-923.

Cuingnet, R., E. Gerardin, J. Tessieras, G. Auzias, S. Lehericy, M. O. Habert, M. Chupin, H. Benali and O. Colliot (2011). "Automatic classification of patients with Alzheimer's disease from structural MRI: a comparison of ten methods using the ADNI database." Neuroimage 56(2): 766-781. 
Du, W., J. Tan, W. Xu, J. Chen and L. Wang (2016). "Association between clusterin gene polymorphism rs11136000 and late-onset Alzheimer's disease susceptibility: A review and meta-analysis of case-control studies." Exp Ther Med12(5): 2915-2927.

Dubey, H., K. Gulati and A. Ray (2018). "Recent studies on cellular and molecular mechanisms in Alzheimer's disease: focus on epigenetic factors and histone deacetylase." Rev Neurosci29(3): 241-260.

Dubois, B., H. H. Feldman, C. Jacova, H. Hampel, J. L. Molinuevo, K. Blennow, S. T. DeKosky, S. Gauthier, D. Selkoe, R. Bateman, S. Cappa, S. Crutch, S. Engelborghs, G. B. Frisoni, N. C. Fox, D. Galasko, M. O. Habert, G. A. Jicha, A. Nordberg, F. Pasquier, G. Rabinovici, P. Robert, C. Rowe, S. Salloway, M. Sarazin, S. Epelbaum, L. C. de Souza, B. Vellas, P. J. Visser, L. Schneider, Y. Stern, P. Scheltens and J. L. Cummings (2014). "Advancing research diagnostic criteria for Alzheimer's disease: the IWG-2 criteria." Lancet Neurol13(6): 614-629.

Farrer, L. A., L. A. Cupples, J. L. Haines, B. Hyman, W. A. Kukull, R. Mayeux, R. H. Myers, M. A. Pericak-Vance, N. Risch and C. M. van Duijn (1997). "Effects of age, sex, and ethnicity on the association between apolipoprotein E genotype and Alzheimer disease. A meta-analysis. APOE and Alzheimer Disease Meta Analysis Consortium." JAMA278(16): 1349-1356.

Franzmeier, N., A. Rubinski, J. Neitzel, M. Ewers and I. Alzheimer's Disease Neuroimaging (2019). "The BIN1 rs744373 SNP is associated with increased tauPET levels and impaired memory." Nat Commun10(1): 1766.

Frederickson, C. J., M. A. Klitenick, W. I. Manton and J. B. Kirkpatrick (1983). "Cytoarchitectonic distribution of zinc in the hippocampus of man and the rat." Brain Res273(2): 335-339.

Hall, J. R., A. R. Wiechmann, L. A. Johnson, M. Edwards, R. C. Barber, R. Cunningham, M. Singh and S. E. O'Bryant (2014). "The Impact of APOE Status on Relationship of Biomarkers of Vascular Risk and Systemic Inflammation to Neuropsychiatric Symptoms in Alzheimer's Disease." J Alzheimers Dis.

Harold, D., R. Abraham, P. Hollingworth, R. Sims, A. Gerrish, M. L. Hamshere, J. S. Pahwa, V. Moskvina, K. Dowzell, A. Williams, N. Jones, C. Thomas, A. Stretton, A. R. Morgan, S. Lovestone, J. Powell, P. Proitsi, M. K. Lupton, C. Brayne, D. C. Rubinsztein, M. Gill, B. Lawlor, A. Lynch, K. Morgan, K. S. Brown, P. A. Passmore, D. Craig, B. McGuinness, S. Todd, C. Holmes, D. Mann, A. D. Smith, S. Love, P. G. Kehoe, J. Hardy, S. Mead, N. Fox, M. Rossor, J. Collinge, W. Maier, F. Jessen, B. Schurmann, R. Heun, H. van den Bussche, I. Heuser, J. Kornhuber, J. Wiltfang, M. Dichgans, L. Frolich, H. Hampel, M. Hull, D. Rujescu, A. M. Goate, J. S. Kauwe, C. Cruchaga, P. Nowotny, J. C. Morris, K. Mayo, K. Sleegers, K. Bettens, S. Engelborghs, P. P. De Deyn, C. Van Broeckhoven, G. Livingston, N. J. Bass, H. Gurling, A. McQuillin, R. Gwilliam, P. Deloukas, A. Al-Chalabi, C. E. Shaw, M. Tsolaki, A. B. Singleton, R. Guerreiro, T. W. Muhleisen, M. M. Nothen, S. Moebus, K. H. Jockel, N. Klopp, H. E. Wichmann, M. M. Carrasquillo, V. S. Pankratz, S. G. Younkin, P. A. Holmans, M. O'Donovan, M. J. Owen and J. Williams (2009). "Genome-wide association study identifies variants at CLU and PICALM associated with Alzheimer's disease." Nat Genet41(10): 1088-1093.

Hohman, T. J., M. E. Koran, T. A. Thornton-Wells and I. Alzheimer's Neuroimaging (2014). "Genetic variation modifies risk for neurodegeneration based on biomarker status." Front Aging_Neurosci6: 183.

Hollingworth, P., D. Harold, R. Sims, A. Gerrish, J. C. Lambert, M. M. Carrasquillo, R. Abraham, M. L. Hamshere, J. S. Pahwa, V. Moskvina, K. Dowzell, N. Jones, A. Stretton, C. Thomas, A. Richards, D. Ivanov, C. Widdowson, J. Chapman, S. Lovestone, J. Powell, P. Proitsi, M. K. Lupton, C. Brayne, D. C. Rubinsztein, M. Gill, B. Lawlor, A. Lynch, K. S. Brown, P. A. Passmore, D. Craig, B. McGuinness, S. Todd, C. Holmes, D. Mann, A. D. Smith, H. Beaumont, D. Warden, G. Wilcock, S. Love, P. G. Kehoe, N. M. Hooper, E. R. Vardy, J. Hardy, S. Mead, N. C. Fox, M. Rossor, J. Collinge, W. Maier, F. Jessen, E. Ruther, B. Schurmann, R. Heun, H. Kolsch, H. van den Bussche, I. Heuser, J. Kornhuber, J. Wiltfang, M. Dichgans, L. Frolich, H. Hampel, J. Gallacher, M. Hull, D. Rujescu, I. Giegling, A. M. Goate, J. S. Kauwe, C. Cruchaga, P. Nowotny, J. C. Morris, K. Mayo, K. Sleegers, K. Bettens, S. Engelborghs, P. P. De Deyn, C. Van Broeckhoven, G. Livingston, N. J. Bass, H. Gurling, A. McQuillin, R. Gwilliam, P. Deloukas, A. Al-Chalabi, C. E. Shaw, M. Tsolaki, A. B. Singleton, R. Guerreiro, T. W. Muhleisen, M. M. Nothen, S. Moebus, K. H. Jockel, N. Klopp, H. E. Wichmann, V. S. Pankratz, S. B. Sando, J. O. Aasly, M. Barcikowska, Z. K. Wszolek, D. W. Dickson, N. R. Graff-Radford, R. C. Petersen, C. M. van Duijn, M. M. Breteler, M. A. Ikram, A. L. DeStefano, A. L. Fitzpatrick, O. Lopez, L. J. Launer, S. Seshadri, C. Berr, D. Campion, J. Epelbaum, J. F. Dartigues, C. Tzourio, A. Alperovitch, M. Lathrop, T. M. Feulner, P. Friedrich, C. Riehle, M. Krawczak, S. Schreiber, M. Mayhaus, S. Nicolhaus, S. Wagenpfeil, S. Steinberg, H. Stefansson, K. Stefansson, J. Snaedal, S. Bjornsson, P. V. Jonsson, V. Chouraki, B. Genier-Boley, M. Hiltunen, H. Soininen, O. Combarros, D. Zelenika, M. Delepine, M. J. Bullido, F. Pasquier, I. Mateo, A. Frank-Garcia, E. Porcellini, O. Hanon, E. Coto, V. Alvarez, P. Bosco, G. Siciliano, M. Mancuso, F. Panza, V. Solfrizzi, B. Nacmias, S. Sorbi, P. Bossu, P. Piccardi, B. Arosio, G. Annoni, D. Seripa, A. Pilotto, E. Scarpini, D. Galimberti, A. Brice, D. Hannequin, F. Licastro, L. Jones, P. A. Holmans, T. Jonsson, M. Riemenschneider, K. Morgan, S. G. Younkin, M. J. Owen, M. O'Donovan, P. Amouyel and J. Williams (2011). "Common variants at ABCA7, MS4A6A/MS4A4E, EPHA1, CD33 and CD2AP are associated with Alzheimer's disease." Nat Genet43(5): 429-435.

Huang, C. W., W. N. Chang, S. H. Huang, C. C. Lui, N. C. Chen, Y. T. Chang, C. C. Lee, C. C. Chang and A. Y. Chang (2013). "Impact of homocysteine on cortical perfusion and cognitive decline in mild Alzheimer's dementia." Eur J Neurol20(8): 1191-1197.

Huang, C. W., S. W. Hsu, S. J. Tsai, N. C. Chen, M. E. Liu, C. C. Lee, S. H. Huang, W. N. Chang, Y. T. Chang, W. C. Tsai and C. C. Chang (2017). "Genetic effect of interleukin-1 beta (C-511T) polymorphism on the structural covariance network and white matter integrity in Alzheimer's disease." J Neuroinflammation $14(1)$ : 12.

Jansen, I. E., J. E. Savage, K. Watanabe, J. Bryois, D. M. Williams, S. Steinberg, J. Sealock, I. K. Karlsson, S. Hagg, L. Athanasiu, N. Voyle, P. Proitsi, A. Witoelar, S. Stringer, D. Aarsland, I. S. Almdahl, F. Andersen, S. Bergh, F. Bettella, S. Bjornsson, A. Braekhus, G. Brathen, C. de Leeuw, R. S. Desikan, S. Djurovic, L.

Dumitrescu, T. Fladby, T. J. Hohman, P. V. Jonsson, S. J. Kiddle, A. Rongve, I. Saltvedt, S. B. Sando, G. Selbaek, M. Shoai, N. G. Skene, J. Snaedal, E. Stordal, I. D. Ulstein, Y. Wang, L. R. White, J. Hardy, J. Hjerling-Leffler, P. F. Sullivan, W. M. van der Flier, R. Dobson, L. K. Davis, H. Stefansson, K. Stefansson, N. L. Pedersen, 
S. Ripke, O. A. Andreassen and D. Posthuma (2019). "Genome-wide meta-analysis identifies new loci and functional pathways influencing Alzheimer's disease risk." Nat Genet51(3): 404-413.

Jiao, B., X. Liu, L. Zhou, M. H. Wang, Y. Zhou, T. Xiao, W. Zhang, R. Sun, M. M. Waye, B. Tang and L. Shen (2015). "Polygenic Analysis of Late-Onset Alzheimer's Disease from Mainland China." PLoS One10(12): e0144898.

Jin, C., W. Li, J. Yuan, W. Xu and Z. Cheng (2012). "Association of the CR1 polymorphism with late-onset Alzheimer's disease in Chinese Han populations: a meta-analysis." Neurosci Lett527(1): 46-49.

Karch, C. M., A. T. Jeng, P. Nowotny, J. Cady, C. Cruchaga and A. M. Goate (2012). "Expression of novel Alzheimer's disease risk genes in control and Alzheimer's disease brains." PLoS One7(11): e50976.

Keenan, B. T., J. M. Shulman, L. B. Chibnik, T. Raj, D. Tran, M. R. Sabuncu, I. Alzheimer's Disease Neuroimaging, A. N. Allen, J. J. Corneveaux, J. A. Hardy, M. J. Huentelman, C. A. Lemere, A. J. Myers, A. Nicholson-Weller, E. M. Reiman, D. A. Evans, D. A. Bennett and P. L. De Jager (2012). "A coding variant in CR1 interacts with APOE-epsilon4 to influence cognitive decline." Hum Mol Genet21(10): 2377-2388.

Lambert, J. C., S. Heath, G. Even, D. Campion, K. Sleegers, M. Hiltunen, O. Combarros, D. Zelenika, M. J. Bullido, B. Tavernier, L. Letenneur, K. Bettens, C. Berr, F. Pasquier, N. Fievet, P. Barberger-Gateau, S. Engelborghs, P. De Deyn, I. Mateo, A. Franck, S. Helisalmi, E. Porcellini, O. Hanon, M. M. de Pancorbo, C. Lendon, C. Dufouil, C. Jaillard, T. Leveillard, V. Alvarez, P. Bosco, M. Mancuso, F. Panza, B. Nacmias, P. Bossu, P. Piccardi, G. Annoni, D. Seripa, D. Galimberti, D. Hannequin, F. Licastro, H. Soininen, K. Ritchie, H. Blanche, J. F. Dartigues, C. Tzourio, I. Gut, C. Van Broeckhoven, A. Alperovitch, M. Lathrop and P. Amouyel (2009).

"Genome-wide association study identifies variants at CLU and CR1 associated with Alzheimer's disease." Nat Genet41(10): 1094-1099.

Lambert, J. C., C. A. Ibrahim-Verbaas, D. Harold, A. C. Naj, R. Sims, C. Bellenguez, A. L. DeStafano, J. C. Bis, G. W. Beecham, B. Grenier-Boley, G. Russo, T. A. Thorton-Wells, N. Jones, A. V. Smith, V. Chouraki, C. Thomas, M. A. Ikram, D. Zelenika, B. N. Vardarajan, Y. Kamatani, C. F. Lin, A. Gerrish, H. Schmidt, B. Kunkle, M. L. Dunstan, A. Ruiz, M. T. Bihoreau, S. H. Choi, C. Reitz, F. Pasquier, C. Cruchaga, D. Craig, N. Amin, C. Berr, O. L. Lopez, P. L. De Jager, V. Deramecourt, J. A. Johnston, D. Evans, S. Lovestone, L. Letenneur, F. J. Moron, D. C. Rubinsztein, G. Eiriksdottir, K. Sleegers, A. M. Goate, N. Fievet, M. W. Huentelman, M. Gill, K. Brown, M. I. Kamboh, L. Keller, P. Barberger-Gateau, B. McGuiness, E. B. Larson, R. Green, A. J. Myers, C. Dufouil, S. Todd, D. Wallon, S. Love, E. Rogaeva, J. Gallacher, P. St George-Hyslop, J. Clarimon, A. Lleo, A. Bayer, D. W. Tsuang, L. Yu, M. Tsolaki, P. Bossu, G. Spalletta, P. Proitsi, J. Collinge, S. Sorbi, F. SanchezGarcia, N. C. Fox, J. Hardy, M. C. Deniz Naranjo, P. Bosco, R. Clarke, C. Brayne, D. Galimberti, M. Mancuso, F. Matthews, I. European Alzheimer's Disease, Genetic, D. Environmental Risk in Alzheimer's, C. Alzheimer's Disease Genetic, H. Cohorts for, E. Aging Research in Genomic, S. Moebus, P. Mecocci, M. Del Zompo, W. Maier, H. Hampel, A. Pilotto, M. Bullido, F. Panza, P. Caffarra, B. Nacmias, J. R. Gilbert, M. Mayhaus, L. Lannefelt, H. Hakonarson, S. Pichler, M. M. Carrasquillo, M. Ingelsson, D. Beekly, V. Alvarez, F. Zou, O. Valladares, S. G. Younkin, E. Coto, K. L. Hamilton-Nelson, W. Gu, C. Razquin, P. Pastor, I. Mateo, M. J. Owen, K. M. Faber, P. V. Jonsson, O. Combarros, M. C. O'Donovan, L. B. Cantwell, H. Soininen, D. Blacker, S. Mead, T. H. Mosley, Jr., D. A. Bennett, T. B. Harris, L. Fratiglioni, C. Holmes, R. F. de Bruijn, P. Passmore, T. J. Montine, K. Bettens, J. I. Rotter, A. Brice, K. Morgan, T. M. Foroud, W. A. Kukull, D. Hannequin, J. F. Powell, M. A. Nalls, K. Ritchie, K. L. Lunetta, J. S. Kauwe, E. Boerwinkle, M. Riemenschneider, M. Boada, M. Hiltuenen, E. R. Martin, R. Schmidt, D. Rujescu, L. S. Wang, J. F. Dartigues, R. Mayeux, C. Tzourio, A. Hofman, M. M. Nothen, C. Graff, B. M. Psaty, L. Jones, J. L. Haines, P. A. Holmans, M. Lathrop, M. A. PericakVance, L. J. Launer, L. A. Farrer, C. M. van Duijn, C. Van Broeckhoven, V. Moskvina, S. Seshadri, J. Williams, G. D. Schellenberg and P. Amouyel (2013). "Metaanalysis of 74,046 individuals identifies 11 new susceptibility loci for Alzheimer's disease." Nat Genet45(12): 1452-1458.

Li, H. L., S. S. Shi, Q. H. Guo, W. Ni, Y. Dong, Y. Liu, Y. M. Sun, W. Bei, S. J. Lu, Z. Hong and Z. Y. Wu (2011). "PICALM and CR1 variants are not associated with sporadic Alzheimer's disease in Chinese patients." J Alzheimers Dis25(1): 111-117.

Mahley, R. W. (1988). "Apolipoprotein E: cholesterol transport protein with expanding role in cell biology." Science240(4852): 622-630.

Martins, C. A., A. Oulhaj, C. A. de Jager and J. H. Williams (2005). "APOE alleles predict the rate of cognitive decline in Alzheimer disease: a nonlinear model." Neurology.65(12): 1888-1893.

Naj, A. C., G. Jun, G. W. Beecham, L. S. Wang, B. N. Vardarajan, J. Buros, P. J. Gallins, J. D. Buxbaum, G. P. Jarvik, P. K. Crane, E. B. Larson, T. D. Bird, B. F. Boeve, N. R. Graff-Radford, P. L. De Jager, D. Evans, J. A. Schneider, M. M. Carrasquillo, N. Ertekin-Taner, S. G. Younkin, C. Cruchaga, J. S. Kauwe, P. Nowotny, P. Kramer, J. Hardy, M. J. Huentelman, A. J. Myers, M. M. Barmada, F. Y. Demirci, C. T. Baldwin, R. C. Green, E. Rogaeva, P. St George-Hyslop, S. E. Arnold, R. Barber, T. Beach, E. H. Bigio, J. D. Bowen, A. Boxer, J. R. Burke, N. J. Cairns, C. S. Carlson, R. M. Carney, S. L. Carroll, H. C. Chui, D. G. Clark, J. Corneveaux, C. W. Cotman, J. L. Cummings, C. DeCarli, S. T. DeKosky, R. Diaz-Arrastia, M. Dick, D. W. Dickson, W. G. Ellis, K. M. Faber, K. B. Fallon, M. R. Farlow, S. Ferris, M. P. Frosch, D. R. Galasko, M. Ganguli, M. Gearing, D. H. Geschwind, B. Ghetti, J. R. Gilbert, S. Gilman, B. Giordani, J. D. Glass, J. H. Growdon, R. L. Hamilton, L. E. Harrell, E. Head, L. S. Honig, C. M. Hulette, B. T. Hyman, G. A. Jicha, L. W. Jin, N. Johnson, J. Karlawish, A. Karydas, J. A. Kaye, R. Kim, E. H. Koo, N. W. Kowall, J. J. Lah, A. I. Levey, A. P. Lieberman, O. L. Lopez, W. J. Mack, D. C. Marson, F. Martiniuk, D. C. Mash, E. Masliah, W. C. McCormick, S. M. McCurry, A. N. McDavid, A. C. McKee, M. Mesulam, B. L. Miller, C. A. Miller, J. W. Miller, J. E. Parisi, D. P. Perl, E. Peskind, R. C. Petersen, W. W. Poon, J. F. Quinn, R. A. Rajbhandary, M. Raskind, B. Reisberg, J. M. Ringman, E. D. Roberson, R. N. Rosenberg, M. Sano, L. S. Schneider, W. Seeley, M. L. Shelanski, M. A. Slifer, C. D. Smith, J. A. Sonnen, S. Spina, R. A. Stern, R. E. Tanzi, J. Q. Trojanowski, J. C. Troncoso, V. M. Van Deerlin, H. V. Vinters, J. P. Vonsattel, S. Weintraub, K. A. Welsh-Bohmer, J. Williamson, R. L. Woltjer, L. B. Cantwell, B. A. Dombroski, D. Beekly, K. L. Lunetta, E. R. Martin, M. I. Kamboh, A. J. Saykin, E. M. Reiman, D. A. Bennett, J. C. Morris, T. J. Montine, A. M. Goate, D. Blacker, D. W. Tsuang, H. Hakonarson, W. A. Kukull, T. M. Foroud, J. L. Haines, R. Mayeux, M. A. Pericak-Vance, L. A. Farrer and G. D. Schellenberg (2011). "Common variants at MS4A4/MS4A6E, CD2AP, CD33 and EPHA1 are associated with late-onset Alzheimer's disease." Nat Genet43(5): 436-441. 
Przezdzik, l., M. Faber, G. Fernandez, C. F. Beckmann and K. V. Haak (2019). "The functional organisation of the hippocampus along its long axis is gradual and predicts recollection." Cortex119: 324-335.

Ramos de Matos, M., C. Ferreira, S. K. Herukka, H. Soininen, A. Janeiro, I. Santana, I. Baldeiras, M. R. Almeida, A. Lleo, O. Dols-Icardo, D. Alcolea, L. Benussi, G. Binetti, A. Paterlini, R. Ghidoni, B. Nacmias, O. Meulenbroek, L. J. C. van Waalwijk van Doorn, H. B. J. Kuiperi, L. Hausner, G. Waldemar, A. H. Simonsen, M. Tsolaki, O. Gkatzima, C. Resende de Oliveira, M. M. Verbeek, J. Clarimon, M. Hiltunen, A. de Mendonca and M. Martins (2018). "Quantitative Genetics Validates Previous Genetic Variants and Identifies Novel Genetic Players Influencing Alzheimer's Disease Cerebrospinal Fluid Biomarkers." J Alzheimers Dis66(2): 639652.

Risacher, S. L., S. Kim, L. Shen, K. Nho, T. Foroud, R. C. Green, R. C. Petersen, C. R. Jack, Jr., P. S. Aisen, R. A. Koeppe, W. J. Jagust, L. M. Shaw, J. Q. Trojanowski, M. W. Weiner and A. J. Saykin (2013). "The role of apolipoprotein E (APOE) genotype in early mild cognitive impairment (E-MCl)." Front Aging Neurosci5: 11.

Segall, J. M., E. A. Allen, R. E. Jung, E. B. Erhardt, S. K. Arja, K. Kiehl and V. D. Calhoun (2012). "Correspondence between structure and function in the human brain at rest." Front Neuroinform6: 10.

Shen, N., B. Chen, Y. Jiang, R. Feng, M. Liao, L. Zhang, F. Li, G. Ma, Z. Chen, B. Zhao, K. Li and G. Liu (2015). "An Updated Analysis with 85,939 Samples Confirms the Association Between CR1 rs6656401 Polymorphism and Alzheimer's Disease." Mol Neurobiol51(3): 1017-1023.

Shulman, J. M., K. Chen, B. T. Keenan, L. B. Chibnik, A. Fleisher, P. Thiyyagura, A. Roontiva, C. McCabe, N. A. Patsopoulos, J. J. Corneveaux, L. Yu, M. J. Huentelman, D. A. Evans, J. A. Schneider, E. M. Reiman, P. L. De Jager and D. A. Bennett (2013). "Genetic susceptibility for Alzheimer disease neuritic plaque pathology." JAMA Neurol70(9): 1150-1157.

Singh, P. P., M. Singh and S. S. Mastana (2006). "APOE distribution in world populations with new data from India and the UK." Ann Hum Biol33(3): 279-308.

Tao, Q. Q., Y. M. Sun, Z. J. Liu, W. Ni, P. Yang, H. L. Li, S. J. Lu and Z. Y. Wu (2014). "A variant within FGF1 is associated with Alzheimer's disease in the Han Chinese population." Am J Med Genet B Neuropsychiatr Genet165B(2): 131-136.

Vogel, J. W., R. La Joie, M. J. Grothe, A. Diaz-Papkovich, A. Doyle, E. Vachon-Presseau, C. Lepage, R. Vos de Wael, R. A. Thomas, Y. Iturria-Medina, B. Bernhardt, G. D. Rabinovici and A. C. Evans (2020). "A molecular gradient along the longitudinal axis of the human hippocampus informs large-scale behavioral systems." Nat Commun11(1): 960.

Wisdom, N. M., J. L. Callahan and K. A. Hawkins (2011). "The effects of apolipoprotein E on non-impaired cognitive functioning: a meta-analysis." Neurobiol Aging32(1): 63-74.

Xiao, Q., Z. J. Liu, S. Tao, Y. M. Sun, D. Jiang, H. L. Li, H. Chen, X. Liu, B. Lapin, C. H. Wang, S. L. Zheng, J. Xu and Z. Y. Wu (2015). "Risk prediction for sporadic Alzheimer's disease using genetic risk score in the Han Chinese population." Oncotarget6(35): 36955-36964.

Zhang, Q., J. T. Yu, Q. X. Zhu, W. Zhang, Z. C. Wu, D. Miao and L. Tan (2010). "Complement receptor 1 polymorphisms and risk of late-onset Alzheimer's disease." Brain Res1348: 216-221.

Zhu, R., X. Liu and Z. He (2018). "Association between CLU gene rs11136000 polymorphism and Alzheimer's disease: an updated meta-analysis." Neurol Sci39(4): 679-689.

\section{Figures}




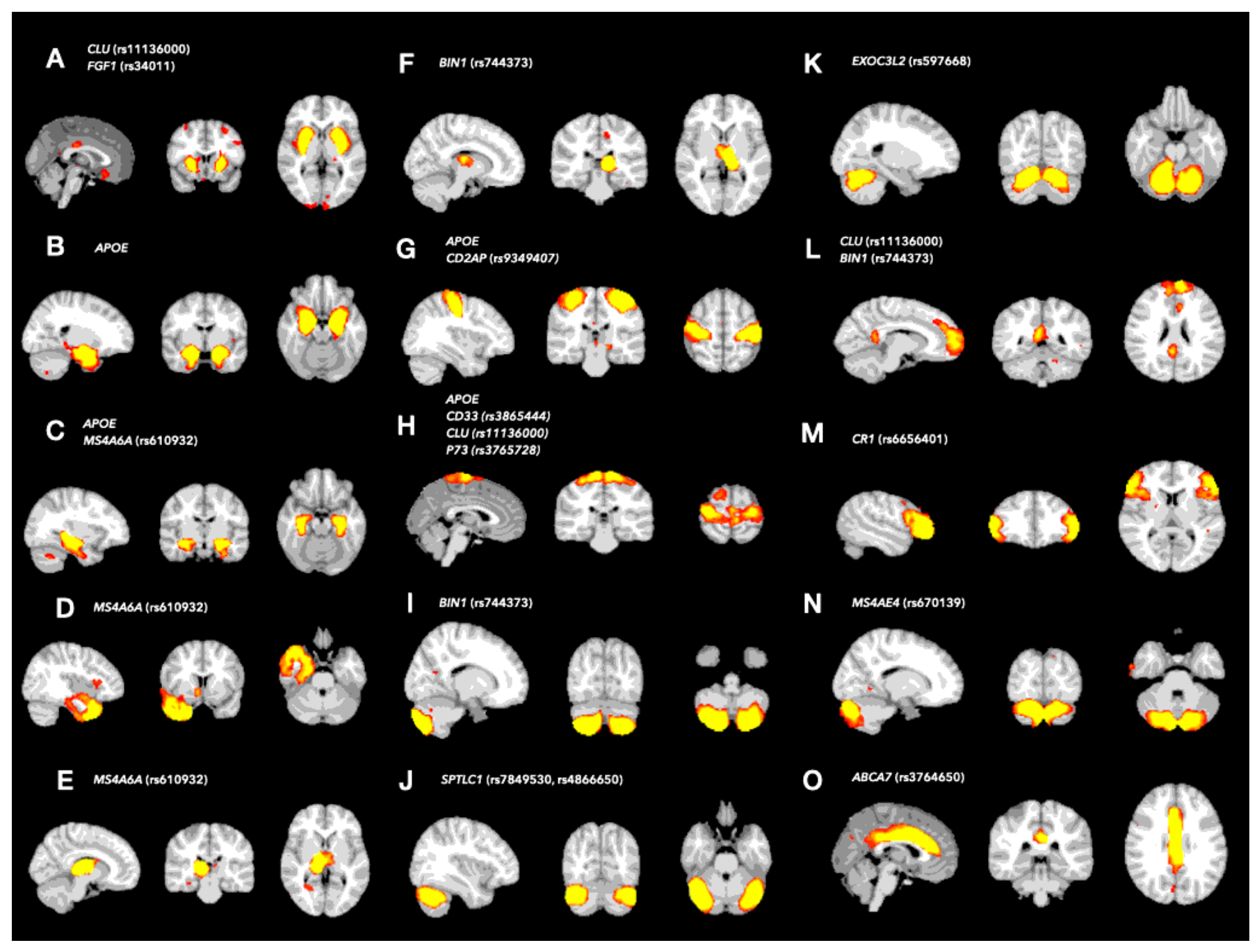

Figure 1

Significant structural covariance network showing group differences in signal intensities using independent component analysis.

\section{Supplementary Files}

This is a list of supplementary files associated with this preprint. Click to download.

- MLC200818SupplementaryTable1.docx

- MLC200818SupplementaryTable2.docx 